\title{
Implementing Sub-sampling Methods for Low-Dose (Scanning) Transmission Electron Microscopy (S/TEM)
}

Nigel D. Browning ${ }^{1,2}$, Andrew Stevens ${ }^{3}$, Libor Kovarik ${ }^{4}$, Andrey Liyu ${ }^{4}$, B. Layla Mehdi ${ }^{1}$, Bryan Stanfill $^{3}$, Sarah Reehl ${ }^{3}$, Lisa Bramer ${ }^{3}$

1. Physical and Computational Science Directorate, PNNL, Richland, WA, USA

2. Materials Science and Engineering, University of Washington, Seattle, WA, USA

3. National Security Directorate, PNNL, Richland, WA, USA

${ }^{5 .}$ Energy and Environmental Directorate, PNNL, Richland, WA, USA

${ }^{6 .}$ Environmental Molecular Sciences Laboratory, PNNL, Richland, WA, USA

In many practical applications of high resolution (scanning) transmission electron microscopy, the resolution obtainable in an image is determined solely by the stability of the sample. This is particularly true in the era of aberration corrected S/TEM where the dose on the sample for the highest resolution images can easily exceed $10^{5}$ electrons/ $\AA^{2}$ during routine operation. This dose sensitivity presents extreme constraints on applications such as 3-D imaging, spectroscopic imaging and in-situ experiments, where prolonged exposure to the beam can result in damage occurring before the experiment can be completed. To ensure that observations are free from damage artifacts, it is therefore essential that images are acquired under conditions where the maximum information can be extracted from the minimum amount of electron dose.

Traditionally low-dose imaging is performed by either acquiring images faster or changing the emission conditions of the gun. However, when there is a change in the emission the microscope alignment changes and it is not always possible to run the automated corrector alignments in low-dose mode to obtain the highest resolution images. Technological limitations are also associated with obtaining images faster; in the case of the TEM low-dose imaging requirements exceed the framerate of the camera, while in STEM the hysteresis in the scan coils limits the overall scanning speed that can be obtained. To overcome some of these limitations it is possible to use a set of image reconstruction methods such as compressive sensing and in-painting [1,2] to reduce the overall number of readouts/pixels in the TEM/STEM images/movies and lower the overall electron dose while at the same time increasing the speed of the image and reducing the overall size of the dataset acquired $[3,4]$.

An example of the use of in-painting to recover pixels in a sub-sampled STEM image is shown in Figure 1. Here the image is sub-sampled after acquisition and recovered to demonstrate that high resolution images typically acquired in a TEM/STEM are over-sampled. Of course, such post-acquisition sub sampling does not decrease the dose on the sample. Setting up the STEM to acquire sub-sampled images directly allows an immediate benefit in terms of the number of pixels sampled (dose, speed and data transfer). Figure 2 shows an image of $\mathrm{CaCo}_{3}$ acquired using a sub-sampling STEM mode of operation [5]. Here the scan proceeds through a pixel hopping mechanism so that a small fraction of the pixels is actually acquired (this also benefits from an increase in speed of acquisition by reducing the number of scan flyback/stabilization procedures during the scan). In this presentation, the use of compressive sensing and in-painting methods to increase the flexibility and speed while decreasing the dose of all modes of TEM/STEM imaging and spectroscopy will be described. The major limitations and the potential benefits of active learning for the acquisition of dynamic movies at vastly increased framerates during in-situ measurements will also be discussed [6]. 
References:

[1] E. J.Candès, J. Romberg, T. Tao, Inform. Theory IEEE Trans. 52, (2006), 489.

[2] D. L. Donoho, Inform. Theory IEEE Trans. 52, (2006), 1289.

[3] A. Stevens, et al, Microscopy 63, (2014), 41.

[4] A. Stevens, et al, Advanced Structural and Chemical Imaging 1, (2015), 10.

[5] L. Kovarik, A. Stevens, A. Liyu, N. D. Browning, Applied Physics Letters 109, 164102 (2016)

[6]This work supported by the Chemical Imaging Initiative (CII) and the Analytics in Motion (AIM) Initiative, Laboratory Directed Research and Development (LDRD) Programs at Pacific Northwest National Laboratory (PNNL). PNNL is a multi-program national laboratory operated by Battelle for the U.S. Department of Energy (DOE) under Contract DE-AC05-76RL01830. A portion of the research was performed using the Environmental Molecular Sciences Laboratory (EMSL), a national scientific user facility sponsored by the Department of Energy's Office of Biological and Environmental Research and located at PNNL.
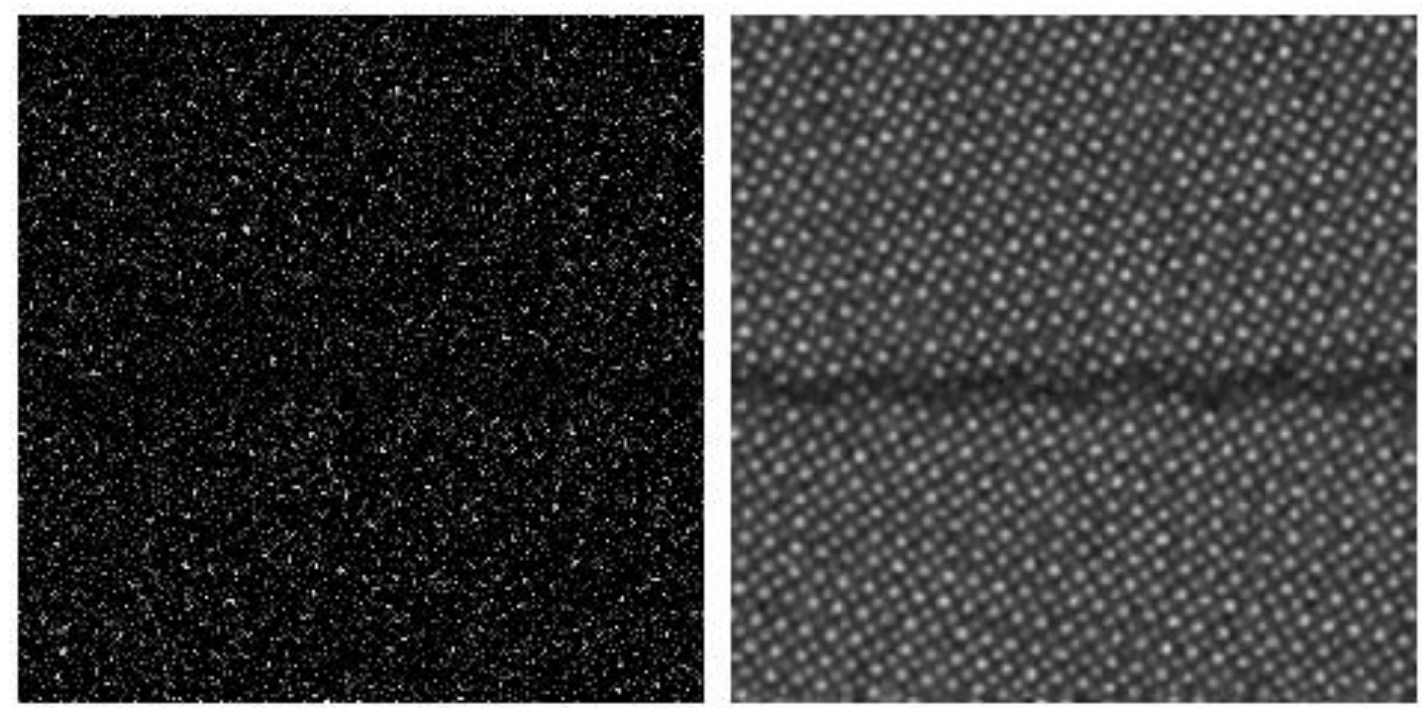

Figure 1. Compressive reconstruction of $\mathrm{SrTiO}_{3}$ grain boundary image with $20 \%$ of the pixels
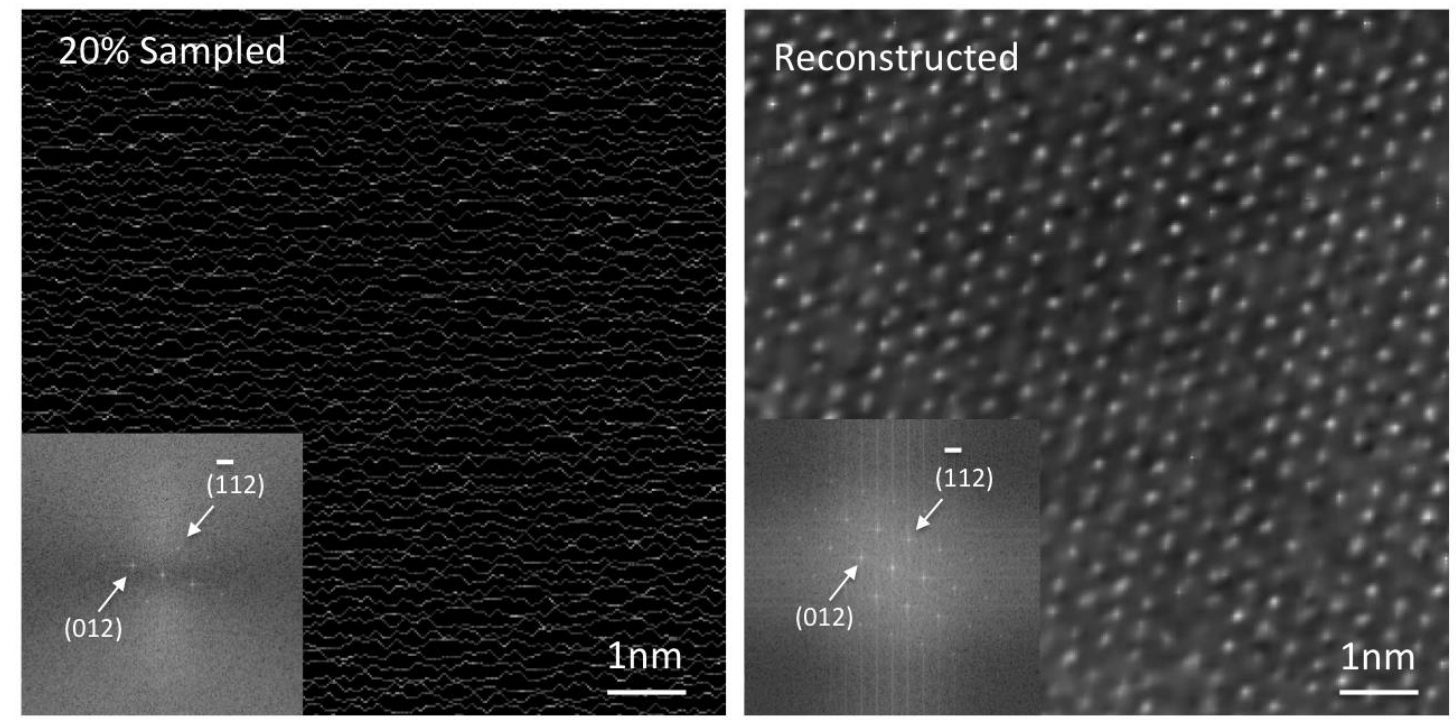

Figure 2. Sparsely sampled and reconstructed images from $\mathrm{CaCO}_{3}$. The sparsely sampled image was acquired with $6 \mathrm{pA}$ probe and dwell time of $31.35 \mu \mathrm{s}$. The pixel size of the image is $0.2719 \mathrm{~A}$, which translates to dose of $3175 \mathrm{e} / \mathrm{A}^{2}$. 